\title{
Cyclic Shift Based Tone Reservation PAPR Reduction Scheme with Embedding Side Information for FBMC-OQAM Systems
}

\author{
Yongpeng Shi ${ }^{1,2}$, Yujie $\mathrm{Xia}^{1,2 *}$, Ya Gao ${ }^{2}$, and Jianhua $\mathrm{Cui}^{2}$ \\ ${ }^{1}$ Henan Key Laboratory for Big Data Processing \& Analytics of Electronic Commerce, Luoyang Normal \\ University, Luoyang, 471022 China \\ [e-mail: syp@lynu.edu.cn] \\ ${ }^{2}$ School of Physics and Electronic Information, Luoyang Normal University \\ Luoyang, 471022 China \\ [e-mail: xyj@lynu.edu.cn] \\ ${ }^{*}$ Corresponding author: Yujie Xia
}

Received June 26, 2020; revised April 15, 2021; revised May 9, 2021; accepted July 10, 2021; published August 31, 2021

\begin{abstract}
The tone reservation (TR) scheme is an attractive method to reduce peak-to-average power ratio (PAPR) in the filter bank multicarrier with offset quadrature amplitude modulation (FBMC-OQAM) systems. However, the high PAPR of FBMC signal will severely degrades system performance. To address this issue, a cyclic shift based TR (CS-TR) scheme with embedding side information (SI) is proposed to reduce the PAPR of FBMC signals. At the transmitter, four candidate signals are first generated based on cyclic shift of the output of inverse discrete Fourier transform (IDFT), and the SI of the selected signal with minimum peak power among the four candidate signals is embedded in sparse symbols with quadrature phase-shift keying constellation. Then, the TR weighted by optimal scaling factor is employed to further reduce PAPR of the selected signal. At the receiver, a reliable SI detector is presented by determining the phase rotation of SI embedding symbols, and the transmitted data blocks can be correctly demodulated according to the detected SI. Simulation results show that the proposed scheme significantly outperforms the existing TR schemes in both PAPR reduction and bit error rate (BER) performances. In addition, the proposed scheme with detected SI can achieve the same BER performance compared to the one with perfect SI.
\end{abstract}

Keywords: peak-to-average power ratio, filter bank multicarrier with offset quadrature amplitude modulation (FBMC-OQAM), tone reservation, side information

This work was supported in part by the Foundation for Young Backbone Teachers in Higher Education Institutions of the Henan Province under Grant 2018GGJS126 and 2020GGJS195, the Key Scientific and Technological Project of the Henan Province under Grant 202102210120 and 212102210553, the Key Scientific Research Program of Higher Education of the Henan Province under Grant 20A510008 and 21A510008, and the Henan key Laboratory for Big Data Processing \& Analytics of Electronic Commerce under Grant 2020-KF-6, China. 


\section{Introduction}

$\mathbf{F}_{\text {ilter bank multicarrier with offset quadrature amplitude modulation (FBMC-OQAM) }}$ signals are based on multicarrier modulation and the adjacent input data blocks are overlap with each other, which result in high peak-to-average power ratio (PAPR) [1-3]. However, high PAPR decreases the efficiency of power amplifier (PA) and leads to non-linear signal distortion at the output of PA [4], which increases bit error rate (BER) and severely degrades the performance of FBMC systems.

Currently, various proposals have been studied to reduce the PAPR of FBMC signals. The available methods presented in the existing works for PAPR reduction of FBMC signals can be broadly classified into two categories One is using conventional FBMC structure with the consideration of overlapping feature of adjacent data blocks. The other is employing new low PAPR FBMC (LP-FBMC) structure based on discrete Fourier transform (DFT) spreading under special identically time-shifted multicarrier (ITSM) condition. Especially, based on the former kind of methods, selected mapping (SLM) [5-10] and partial transmit sequences (PTS) [11-19] schemes were proposed with requiring side information (SI) transmission. Although they can achieve good PAPR performance, the complexity is usually high and the correct transmission of SI is critical for data demodulation at the receiver. To avoid SI transmission, some PAPR reduction methods such as active constellation extension (ACE), clipping and tone reservation (TR) were discussed. The authors in [20-23] presented an ACE-based method to improve PAPR performance at the cost of increasing the transmitting power. For the clipping method in [24], the BER performance degrades because of signal distortion with hard clipping. The TR scheme in [25-28] is an attractive technique without signal distortion and SI transmission, which reserved some subcarriers as peak reduction tones (PRTs) and generated peak reduction signal to mitigate PAPR. Conventional clipping based TR (CC-TR) [25], sliding window tone reservation (SW-TR) [26] and multi-block TR (MB-TR) [27] schemes were presented to reduce PAPR with large number of iterations required. In order to improve convergence speed, the overlapping scaling TR (OS-TR) [28] was designed by weighting an optimal scaling factor to approximate the peak-cancelling signal, which effectively reduced PAPR with small number of iterations. Nevertheless, the PAPR is still high and the BER performance degrades for FBMC systems. Another hybrid schemes were presented in [29] and [30] by taking advantage of PTS and TR, but the main drawbacks are the SI burden and high complexity.

As for the second kind of methods, a LP-FBMC method was discussed in [31] to generate four candidate signals and transmitted the one with the minimum PAPR signal. In [32], a PAPR reduction scheme was extended to Alamouti-coded LP-FBMC systems. However, the LP-FBMC method needs to transmit two bits SI to indicate the selected signal. To avoid SI burden, an improved PAPR reduction scheme was presented in [33] by embedding SI in LP-FBMC (SI LP-FBMC) signals. Unfortunately, if the number of SI embedded symbols with quadrature phase-shift keying (QPSK) constellation is small, the SI detection performance is poor by taking the fourth power operation. From the above discussion, we observe that it is different between the LP-FBMC structure and the conventional FBMC structure. Moreover, the PAPR of LP-FBMC signal is still high because only four candidate signals are used.

In order to achieve good PAPR reduction and improve BER performance, we devised a cyclic shift based TR scheme, denoted by CS-TR, with SI embedded for FBMC signals. In our proposed scheme, candidate signals selection and weighted TR are joint optimization by 
making full use of the overlapped structure of FBMC signals. The main contributions of this paper are summarized in three folds as follows.

(1) Inspired by the candidate signals selection of LP-FBMC scheme [31], we design four candidate signals based on cyclic delay of the output of inverse discrete Fourier transform (IDFT), and embed SI into small number of SI subcarriers with QPSK modulation. The selected signal with minimum power is then handled by weighted TR method to further reduce PAPR with fast convergence speed at the transmitter.

(2) We propose a simple and reliable SI detector at the receiver. The SI is determined by measuring the phase rotation of sparse SI embedding symbols. Then, the transmitted data blocks are correctly recovered with detected SI.

(3) We evaluate the proposed CS-TR scheme by simulations. We confirm that the presented method can offer satisfied PAPR reduction and BER performance for FBMC signals.

The rest of this paper is organized as follows. FBMC/OQAM signal model is shortly introduced in Section 2. In Section 3, cyclic-shift based candidate signals are generated and a novel CS-TR PAPR reduction scheme with embedding SI is proposed at the transmitter, and then the effective SI detector is presented at the receiver. Section 4 analyzes computational complexity and Section 5 shows the simulation results and performance comparisons. Finally, the whole paper is concluded in Section 6.

\section{FBMC/OQAM Signal Model}

In a typical FBMC/OQAM system, the complex symbols are modulated based on OQAM, and the $M$ complex data blocks with $N$ subcarriers at the transmitter can be written as

$$
\mathbf{C}=\left[\mathbf{C}_{0}, \cdots, \mathbf{C}_{m}, \cdots, \mathbf{C}_{M-1}\right]
$$

where $\mathbf{C}_{m}$ is the $m$ th complex data block which is defined as $\mathbf{C}_{m}=\left[C_{m, 0}, \cdots, C_{m, n}, \cdots, C_{m, N-1}\right]^{T}$ with $C_{m, n}=a_{m, n}+j b_{m, n}$ for $0 \leq m \leq M-1,0 \leq n \leq N-1$. The real part $a_{m, n}$ and the imaginary part $b_{m, n}$ of $C_{m, n}$ pass through a bank of prototype filter $p(t)$. The length of $p(t)$ is $K T$ where $K$ is oversampling factor and $T$ denotes symbol duration. To reduce the complexity of FBMC modulator, IDFT and polyphase network (PPN) techniques are employed for FBMC transmitter [31-34]. Fig. 1 shows a typical IDFT-PPN structure of FBMC/OQAM transmitter.

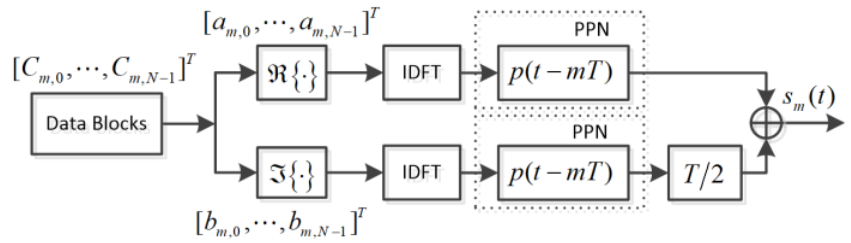

Fig. 1. A typical IDFT-PPN structure of FBMC-OQAM transmitter

As shown in Fig. 1, the baseband signal of the $m$ th data block for $m T \leq t \leq(m+K+1 / 2) T$ in time domain is

$$
\begin{aligned}
S_{m}(t) & =(-1)^{m} \sum_{n=0}^{N-1}\left\{j^{n} a_{m, n} p(t-m T) e^{j n 2 \pi t / T}+j^{n+1} b_{m, n} p(t-m T-T / 2) e^{j 2 \pi n(t-T / 2) / T}\right\} \\
& =\sum_{n=0}^{N-1}\left\{a_{m, n} g_{m, n}(t)+j b_{m, n} g_{m, n}(t-T / 2)\right\}
\end{aligned}
$$

where $g_{m, n}(t)=(-1)^{m} j^{n} p(t-m T) e^{j n 2 \pi t / T}$. 
For all $M$ data blocks, the FBMC signal for $0 \leq t \leq(M+K-1 / 2) T$ in time domain is

$$
s(t)=\sum_{m=0}^{M-1} s_{m}(t)=\sum_{m=0}^{M-1} \sum_{n=0}^{N-1}\left\{a_{m, n} g_{m, n}(t)+j b_{m, n} g_{m, n}(t-T / 2)\right\}
$$

Since the FBMC signals are overlapped with adjacent data blocks caused by the prototype filter $p(t)$, the PAPR for $s(t)$ on the interval $[v T,(v+1) T]$ for $v \in[0, M+K+1]$ is defined as

$$
\operatorname{PAPR}\left[s_{v}(t)\right]=\frac{\max _{v T \leq t \leq(v+1) T}|s(t)|^{2}}{E\left[|s(t)|^{2}\right]}
$$

where $E\left[|s(t)|^{2}\right]$ represents the average power of transmitted FBMC signals.

At the receiver, when the received signal is $r(t)$, the estimations of $a_{m, k}$ and $b_{m, k}$ for the $m$ th block over the $k$ th subcarrier, denoted by $\hat{a}_{m, k}$ and $\hat{b}_{m, k}$ respectively, are calculated as follows

$$
\begin{aligned}
& \hat{a}_{m, k}=\Re\left[\int_{-\infty}^{+\infty} r(t) g_{m, k}^{*}(t) d t\right], \\
& \hat{b}_{m, k}=\Re\left[(-j) \int_{-\infty}^{+\infty} r(t) g_{m, k}^{*}(t-T / 2) d t\right]
\end{aligned}
$$

where $\mathfrak{R}[\cdot]$ is the real operation. Consequently, the estimation of the $m$ th input data symbol, denoted by $\hat{C}_{m, k}$, can be obtained as

$$
\hat{C}_{m, k}=\hat{a}_{m, k}+j \hat{b}_{m, k}
$$

\section{Proposed PAPR Reduction Scheme for FBMC Signals}

In this section, a CS-TR scheme with embedding SI is discussed to reduce PAPR of FBMC signals. The PAPR reduction of the proposed scheme is accomplished by three steps. Firstly, four candidate signals are generated based on the cyclic shift of the output of IDFT. After using sequential optimization procedure to deal with each data block, the one with minimum peak power is selected from the four candidate signals. Then, the SI of the selected signal is embedded into some sparse symbols with QPSK modulation. Secondly, TR weighted by optimal scaling factor method is employed on the selected signal to further reduce the PAPR. Finally, the reliable SI detector is presented by measuring the phase rotation of the SI embedding symbols at the receiver.

\subsection{Cyclic Shift-based FBMC Signals}

The structure of cyclic shift based FBMC-OQAM signals is shown in Fig. 2. The real part $a_{m, n}$ and the imaginary part $b_{m, n}$ of the $m$ th input symbol $C_{m, n}$ are processed by IDFT and the outputs are denoted as $y_{m}^{(1)}(t)$ and $y_{m}^{(3)}(t)$. After performing a cyclic shift $T / 2$ of the IDFT outputs, we obtain the following two signals $y_{m}^{(2)}(t)=y_{m}^{(1)}(t-T / 2)$ and $y_{m}^{(4)}(t)=y_{m}^{(3)}(t-T / 2)$.

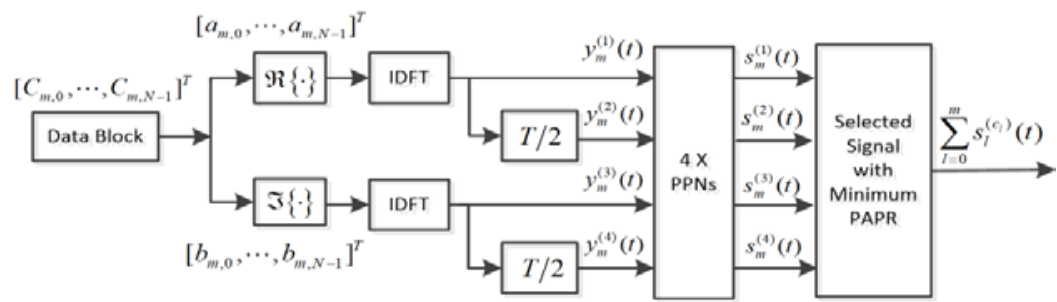

Fig 2. Architecture of cyclic shift-based FBMC-OQAM signals 
The four signals $y_{m}^{(1)}(t), y_{m}^{(2)}(t), y_{m}^{(3)}(t)$ and $y_{m}^{(4)}(t)$ pass through prototype filter $p(t)$ with PPN, and the four candidate signals $s_{m}^{(c)}(t)$ for $c=1,2,3,4$ can be expressed as

$$
\begin{aligned}
& s_{m}^{(1)}(t)=y_{m}^{(1)}(t)+y_{m}^{(3)}(t)=(-1)^{m} \sum_{n=0}^{N-1}\left[j^{n} a_{m, n} p(t-m T) e^{j \frac{2 \pi}{T} t}+j^{n+1} b_{m, n} p(t-m T-T / 2) e^{j \frac{2 \pi}{T} n(t-T / 2)}\right] \\
& s_{m}^{(2)}(t)=y_{m}^{(1)}(t)+y_{m}^{(4)}(t)=(-1)^{m} \sum_{n=0}^{N-1}\left[j^{n} a_{m, n} p(t-m T) e^{j n \frac{2 \pi}{T} t}+j^{n+1} b_{m, n} p(t-m T-T / 2) e^{j \frac{2 \pi}{T} n(t-T)}\right] \\
& s_{m}^{(3)}(t)=y_{m}^{(2)}(t)+y_{m}^{(3)}(t)=(-1)^{m} \sum_{n=0}^{N-1}\left[j^{n} a_{m, n} p(t-m T) e^{j n \frac{2 \pi}{T}(t-T / 2)}+j^{n+1} b_{m, n} p(t-m T-T / 2) e^{j \frac{2 \pi}{T} n(t-T / 2)}\right] \\
& s_{m}^{(4)}(t)=y_{m}^{(2)}(t)+y_{m}^{(4)}(t)=(-1)^{m} \sum_{n=0}^{N-1}\left[j^{n} a_{m, n} p(t-m T) e^{j n \frac{2 \pi}{T}(t-T / 2)}+j^{n+1} b_{m, n} p(t-m T-T / 2) e^{j \frac{2 \pi}{T} n(t-T)}\right]
\end{aligned}
$$

Based on $e^{j n \frac{2 \pi}{T}(t+T / 2)}=(-1)^{n} e^{j n \frac{2 \pi}{T} t}$, the terms of IDFT outputs with time shift $T / 2$ lead to phase rotation $e^{j \pi n}=(-1)^{n}$ in FBMC modulation stage. Therefore, the four candidate signals of (8)-(11) can be rewritten as

$$
\begin{aligned}
s_{m}^{(1)}(t) & =\sum_{n=0}^{N-1}\left\{a_{m, n} g_{m, n}(t)+j b_{m, n} g_{m, n}(t-T / 2)\right\} \\
s_{m}^{(2)}(t) & =\sum_{n=0}^{N-1}\left\{a_{m, n} g_{m, n}(t)+j(-1)^{n} b_{m, n} g_{m, n}(t-T / 2)\right\} \\
s_{m}^{(3)}(t) & =\sum_{n=0}^{N-1}\left\{(-1)^{n} a_{m, n} g_{m, n}(t)+j b_{m, n} g_{m, n}(t-T / 2)\right\} \\
s_{m}^{(4)}(t) & =\sum_{n=0}^{N-1}\left\{(-1)^{n} a_{m, n} g_{m, n}(t)+j(-1)^{n} b_{m, n} g_{m, n}(t-T / 2)\right\}
\end{aligned}
$$

For ease of presentation, we rewrite $\left\{s_{m}^{(c)}(t), c=1,2,3,4\right\}$ in equations (12)-(15) as follows:

$$
S_{m}^{(c)}(t)=\left\{\begin{array}{l}
\sum_{n=0}^{N-1}\left\{a_{m, n} g_{m, n}(t)+j b_{m, n} g_{m, n}(t-T / 2)\right\}, c=1 \\
\sum_{n=0}^{N-1}\left\{a_{m, n} g_{m, n}(t)+j(-1)^{n} b_{m, n} g_{m, n}(t-T / 2)\right\}, c=2 \\
\sum_{n=0}^{N-1}\left\{(-1)^{n} a_{m, n} g_{m, n}(t)+j b_{m, n} g_{m, n}(t-T / 2)\right\}, c=3 \\
\sum_{n=0}^{N-1}\left\{(-1)^{n} a_{m, n} g_{m, n}(t)+j(-1)^{n} b_{m, n} g_{m, n}(t-T / 2)\right\}, c=4
\end{array}\right.
$$

Considering the overlapped feature of FBMC signal, we adopt a sequential optimization procedure to deal with each data block to reduce PAPR. Let $\bar{s}_{m}(t)$ denote the concatenated waveform up to the $m$ th block, we have $\bar{s}_{m}(t)=\bar{s}_{m-1}(t)+s_{m}^{\left(c_{m}\right)}(t)$ with $\bar{s}_{-1}(t)=0$ where $c_{m}$ is the selected SI index of the $m$ th data block among the four candidate signals $\left\{s_{m}^{(c)}(t), c=1,2,3,4\right\}$. Therefore, $c_{m}$ can be determined by

$$
C_{m}=\left\{\begin{array}{l}
\arg \min \left\{\max _{c \in\{1,2,3,4\}}\left|s_{0 \leq t \leq(K+1 / 2) T}^{(c)}(t)\right|^{2}\right\}, m=0 \\
\underset{c \in\{1,2,3,4\}}{\arg \min }\left\{\max _{m T \leq t \leq(m+K+1 / 2) T}\left|\bar{S}_{m-1}(t)+s_{m}^{(c)}(t)\right|^{2}\right\}, m \neq 0
\end{array}\right.
$$

Obviously, $s_{m}^{\left(c_{m}\right)}(t)$ is the selected signal of the $m$ th block in terms of minimum peak power of $\left\{\bar{s}_{m-1}(t)+s_{m}^{(c)}(t), c=1,2,3,4\right\}$ for $m T \leq t \leq(m+K+1 / 2) T$. The transmitted signal for all $M$ data 
blocks in time domain is

$$
s(t)=\sum_{m=0, c_{m} \in\{1,2,3,4\}}^{M-1} s_{m}^{\left(c_{m}\right)}(t), 0 \leq t \leq(M+K-1 / 2) T
$$

Assuming that the wireless channel is ideal and the effect of additive white Gaussian noise (AWGN) is neglected, the received signal $s(t)$ can be demodulated by using (5) and (6). For FBMC/OQAM system, the prototype filter $p(t)$ satisfies the perfect reconstruction condition with $\int_{-\infty}^{+\infty} g_{k, n}(t) g_{k, n}^{*}(t) d t=1$ [3]. Therefore, the estimations $\hat{a}_{n, m}$ and $\hat{b}_{k, n}$ of the $m$ th block over the $k$ th subcarrier are given as

$$
\begin{aligned}
\hat{a}_{m, k} & =\Re\left\{\int_{-\infty}^{+\infty} s(t) g_{m, k}^{*}(t) d t\right\} \\
& =\Re\left\{\int_{-\infty}^{+\infty}\left[\sum_{l=0, l \neq m}^{M-1} s_{l}^{\left(c_{l}\right)}(t)+s_{m}^{\left(c_{m}\right)}(t)\right] g_{m, k}^{*}(t) d t\right\} \\
& =\left\{\begin{array}{l}
a_{m, k}, \quad c_{m}=1,2 \\
(-1)^{n} a_{m, k}, c_{m}=3,4
\end{array}\right. \\
\hat{b}_{m, k} & =\Re\left\{(-j) \int_{-\infty}^{+\infty} s(t) g_{m, k}^{*}(t-T / 2) d t\right\} \\
& =\Re\left\{(-j) \int_{-\infty}^{+\infty}\left[\sum_{l=0, l \neq m}^{M-1} s_{l}^{\left(c_{l}\right)}(t)+s_{m}^{\left(c_{m}\right)}(t)\right] g_{m, k}^{*}(t-T / 2) d t\right\} \\
& =\left\{\begin{array}{lc}
b_{m, k}, & c_{m}=1,3 \\
(-1)^{n} b_{m, k}, c_{m}=2,4
\end{array}\right.
\end{aligned}
$$

Substituting (19) and (20) into (7), the estimated demodulated symbol $\hat{C}_{m, k}$ of the $m$ th block on the $k$ th subcarrier is given as

$$
\hat{C}_{m, k}=\hat{a}_{m, k}+j \hat{b}_{m, k}=\left\{\begin{array}{l}
a_{m, k}+j b_{m, k}, \quad c_{m}=1 \\
a_{m, k}+j(-1)^{n} b_{m, k}, \quad c_{m}=2 \\
(-1)^{n} a_{m, k}+j b_{m, k}, \quad c_{m}=3 \\
(-1)^{n} a_{m, k}+j(-1)^{n} b_{m, k}, \quad c_{m}=4
\end{array}\right.
$$

Thus, the input data symbols $C_{m, k}$ will be successfully recovered from the estimated demodulated symbols $\hat{C}_{m, k}$, when the SI index $\hat{c}$ of selected signal is correctly determined. The recovered data symbols $D_{m, k}$ is calculated as

$$
D_{m, k}= \begin{cases}\hat{a}_{m, k}+j \hat{b}_{m, k} & c_{m}=1 \\ \hat{a}_{m, k}+j(-1)^{n} \hat{b}_{m, k}, & c_{m}=2 \\ (-1)^{n} \hat{a}_{m, k}+j \hat{b}_{m, k}, & c_{m}=3 \\ (-1)^{n} \hat{a}_{m, k}+j(-1)^{n} \hat{a}_{m, k}, & c_{m}=4\end{cases}
$$

\subsection{Proposed CS-TR PAPR Reduction Scheme with SI Embedded}

When one symbol $C_{m, n}=(1+j) / \sqrt{2}$ of the $m$ th block is delicately used on the $n$th odd subcarrier (index of $n$ is odd), the demodulated symbol $\hat{C}_{m, n}$ in (22) is given as 


$$
\hat{C}_{m, n}= \begin{cases}e^{j \pi / 4}, & c_{m}=1 \\ e^{-j \pi / 4}, & c_{m}=2 \\ e^{j 3 \pi / 4}, & c_{m}=3 \\ e^{-j 3 \pi / 4}, & c_{m}=4\end{cases}
$$

As shown in (23), for different SI $c_{m}$, the demodulated symbol $\hat{C}_{m, n}$ on odd subcarrier has different phase rotations, which means that we can embed the SI into $N_{P}$ sparse symbols on odd subcarriers with QPSK constellation. Consequently, the SI detection problem can be solved by searching the phase rotation of sparse symbols at the receiver.

Based on the above discussion, a new CS-TR scheme is proposed to reduce PAPR for FBMC signal and the block diagram of the proposed scheme at the transmitter is depicted in Fig. 3. For FBMC/OQAM systems, the total $N$ subcarriers are grouped into $N_{R}$ PRTs for peak reduction, $N_{P}$ sparse odd subcarriers for embedding SI and $N_{D}$ data subcarriers for data transmission where $N=N_{D}+N_{P}+N_{R}$. The indices of $N_{R}$ PRTs, $N_{P}$ SI subcarriers and $N_{D}$ data subcarriers are denoted by the sets $J_{R}, J_{P}$ and $J_{D}$, respectively. Note that the number of subcarriers $N_{P}$ is usually small. The $m$ th input data block $\mathbf{C}_{m}=\left[C_{m, 0}, \cdots, C_{m, n}, \cdots, C_{m, N-1}\right]^{T}$, consisting of $N_{R}$ peak reduction symbols $\left\{F_{m, n}, n \in J_{R}\right\}$ with $\left\{F_{m, n} \equiv 0, n \notin J_{R}\right\}, N_{P}$ SI embedded symbols $\left\{C_{m, n}^{P}, n \in J_{P}\right\}$ with $\left\{C_{m, n}^{P} \equiv 0, n \notin J_{P}\right\}$ and $N_{D}$ data symbols $\left\{C_{m, n}^{D}, n \in J_{D}\right\}$ with $\left\{C_{m, n}^{D} \equiv 0, n \notin J_{D}\right\}$, can be represented as

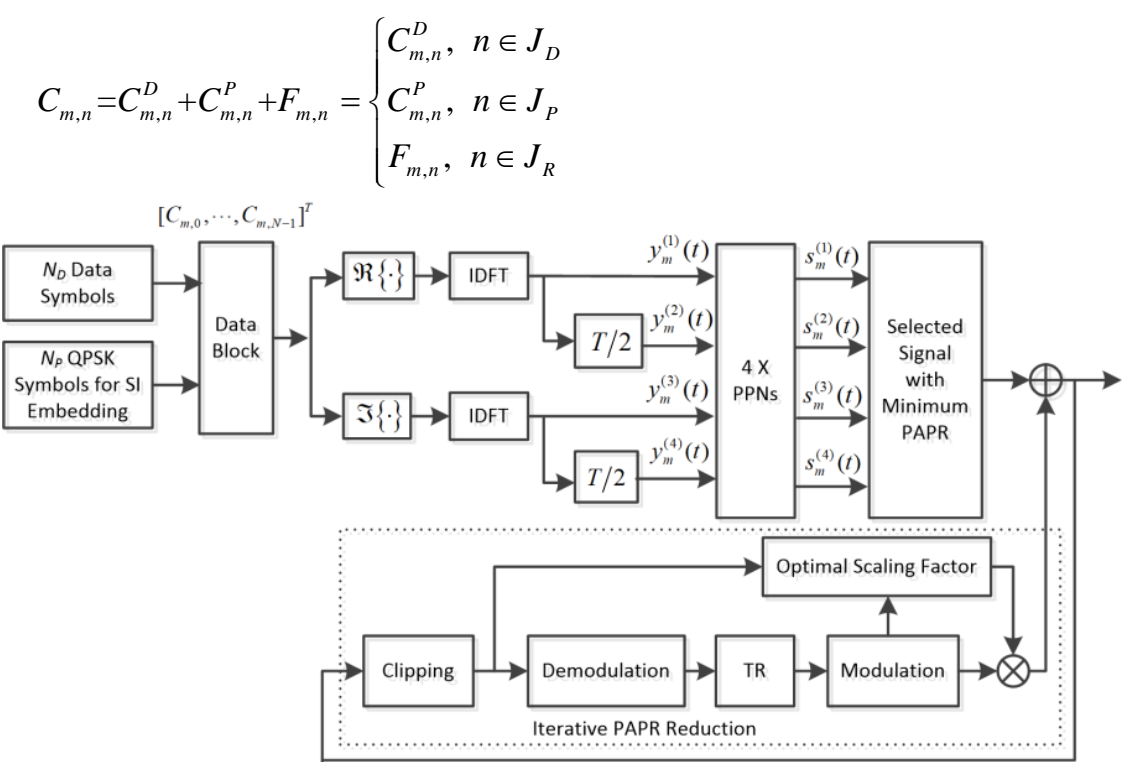

Fig 3. Block diagram of the transmitter of the proposed CS-TR PAPR reduction scheme

As shown in Fig. 3, for the $m$ th input data block, when the $N_{P}$ sparse symbols $\left\{C_{m, n}^{P}=(1+j) / \sqrt{2}, n \in J_{P}\right\}$ for embedding SI are inserted into the $N_{D}$ data symbols $\left\{C_{m, n}^{D}, n \in J_{D}\right\}$ with $\left\{F_{m, n}=0, n \in J_{R}\right\}$, the four candidate signals $\left\{s_{m}^{(c)}(t), c=1,2,3,4\right\}$ are generated according to (8)-(11). Suppose that $s_{m}^{\left(c_{m}\right)}(t)$ is the selected signal of the $m$ th block with minimum peak power 
among the four candidate signals and $c_{m}$ is the SI index of selected signal in terms of (17), the desired FBMC signal $s(t)=\sum_{m=0}^{M-1} s_{m}^{\left(c_{m}\right)}(t)$ is obtained by summing all $M$ data blocks.

In order to further reduce PAPR of $s(t)$, an iterative TR method is employed by weighting scaling factors to $s(t)$. The clipping ratio (CR) for TR is set to $\lambda$ and the peak clipped signal $\hat{s}^{(i)}(t)$ at the ith iteration is

$$
\hat{s}^{(i)}(t)= \begin{cases}s^{(i)}(t), & \left|s^{(i)}(t)\right| \leq A \\ A e^{j \theta^{(i)}} & \left|s^{(i)}(t)\right|>A\end{cases}
$$

where $s^{(i)}(t)$ is the peak reduced signal at the ith iteration, $\theta^{(i)}$ is the phase of $s^{(i)}(t)$ and $A$ is the clipping threshold with $A^{2}=\lambda P^{(i)}$ where $P^{(i)}$ is the average power of $s^{(i)}(t)$. The clipped noise in time domain can be expressed as

$$
f^{(i)}(t)=\hat{s}^{(i)}(t)-s^{(i)}(t), 0 \leq t \leq(M+K-1 / 2) T
$$

Using (5) and (6), we can obtain the modulated symbol $\left\{\hat{F}_{m, n}^{(i)}, 0 \leq m \leq M-1\right\}$ of clipped noise $f^{(i)}(t)$. Except for all $N_{R}$ PRTs, set $\left\{\hat{F}_{m, n}^{(i)}\right\}$ to zero and the modulated symbol of clipped noise at the ith iteration is given by

$$
F_{m, n}^{(i)}=\left\{\begin{array}{l}
\hat{F}_{m, n}^{(i)}, n \in J_{R} \\
0, \quad n \notin J_{R}
\end{array}\right.
$$

Then, we modulate $\left\{F_{m, n}^{(i)}, 0 \leq m \leq M-1\right\}$ using (3) to obtain peak-cancelling signal $\hat{f}^{(i)}(t)$ with $0 \leq t \leq(M+K-1 / 2) T$. Considering the overlapped feature of $\hat{f}^{(i)}(t)$, let $\bar{f}_{m}^{(i)}(t)$ be the overlapped time domain signal of $\hat{f}^{(i)}(t)$ ranging from $m T$ to $(m+K+1 / 2) T$. To make $\hat{f}^{(i)}(t)$ as much as possible to approximate $f^{(i)}(t)$ for PAPRA reduction, we have

$$
\min _{\rho_{0}^{(i)}, \ldots, \rho_{M-1}^{(i)}}\left\{\sum_{t=0}^{(M+K-1 / 2) T}\left|\sum_{m=0}^{M-1} \rho_{m}^{(i)} \bar{f}_{m}^{(i)}(t)-f^{(i)}(t)\right|^{2}\right\}
$$

where $\left\{\rho_{m}^{(i)}, m=0,1, \cdots M-1\right\}$ are scaling factors at the ith iteration. The optimal $\rho_{m}^{(i)}$ in (28) is calculated by least squares method [28], which is

$$
\rho_{m}^{(i)}=\left\{\begin{array}{l}
\frac{\sum_{t \in S_{0}}\left|\bar{f}_{f^{(i)}}(t)\right|\left|f_{0}^{(i)}(t)\right|}{\sum_{t \in S_{0}}\left|\bar{f}_{0}^{(i)}(t)\right|^{2}}, m=0 \\
\frac{\sum_{t \in S_{m}} \bar{f}_{m}^{(i)}(t)|| f_{m}^{(i)}(t)-\sum_{k=0}^{m-1} \rho_{k}^{(i)} \bar{f}_{k}^{(i)}(t) \mid}{\sum_{t \in S_{m}}\left|\bar{f}_{m}^{(i)}(t)\right|^{2}}, 1 \leq m \leq M-1
\end{array}\right.
$$

where $S_{m}=\left\{t /\left|f_{m}^{(i)}(t)\right|>0, m T \leq t \leq(m+K+1 / 2) T\right\}$.

Thus, the peak-cancelling signal at the ith iteration is reconstructed as

$$
z^{(i)}(t)=\sum_{m=0}^{M-1} \rho_{m}^{(i)} \hat{f}_{m}^{(i)}(t)
$$

where $\hat{f}_{m}^{(i)}(t)$ is time domain signal of $\hat{F}_{m, n}^{(i)}$ with $m T \leq t \leq(m+K+1 / 2) T$. Therefore, the peak reduced signal in time domain at the $(i+1)$ th iteration is 


$$
s^{(i+1)}(t)=s^{(i)}(t)+z^{(i)}(t)
$$

The details of the proposed PAPR reduction scheme are illustrated in algorithm 1.

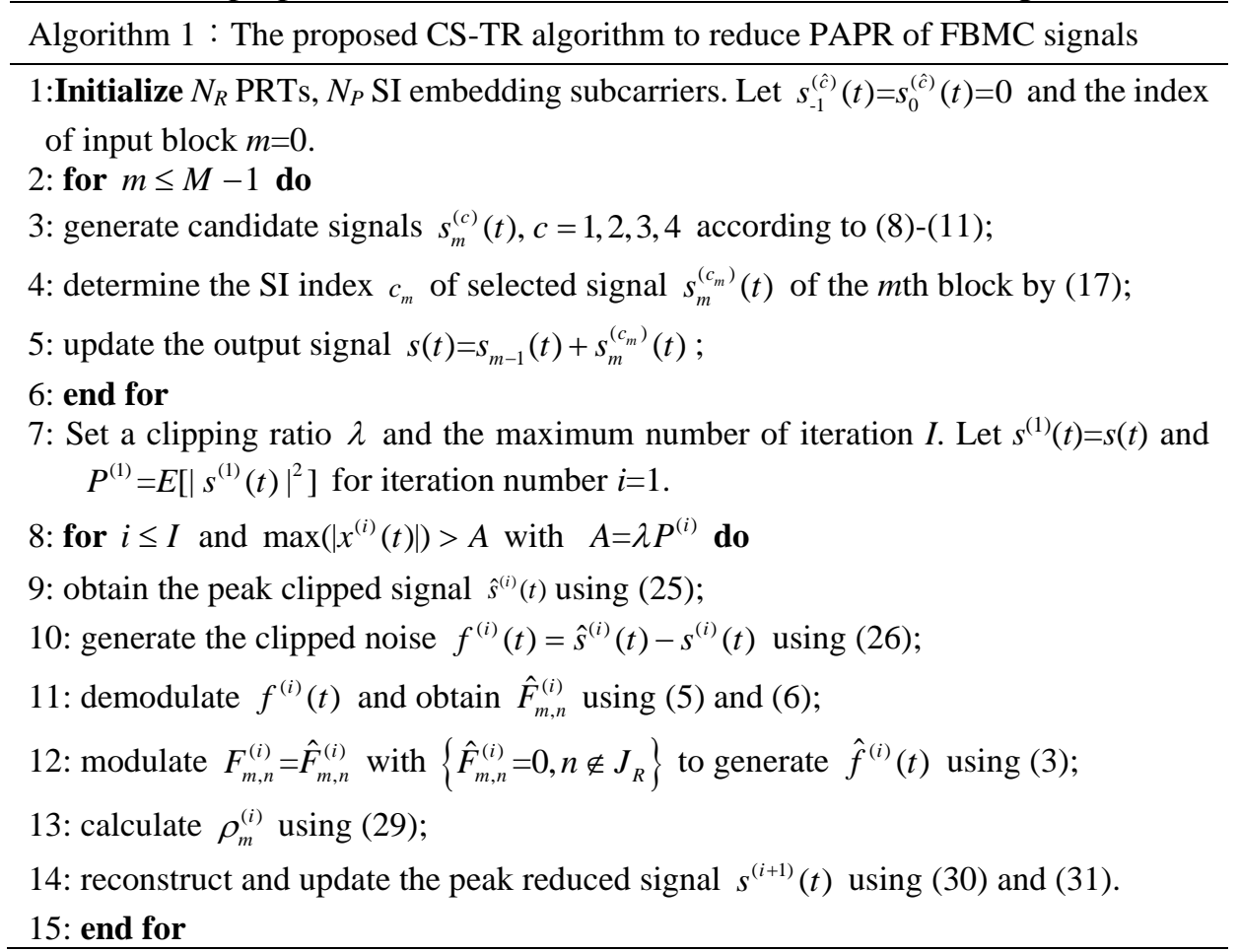

\subsection{Proposed SI Detector}

At the receiver, the PPN and DFT are performed and the received symbols on the PRTs are discarded. Note that the embedding SI symbols $\left\{C_{m, n}^{P}=(1+j) / \sqrt{2}, n \in J_{P}\right\}$ are known at the receiver. Let $\left\{\hat{Y}_{m, n}^{P}, n \in J_{P}\right\}$ and $\left\{\hat{C}_{m, n}^{P}, n \in J_{P}\right\}$ denote the received symbols and the demodulated symbols on SI subcarriers with perfectly equalization of the wireless channel [35][36], the received symbol $\hat{Y}_{m, n}^{P}$ on SI subcarriers can be expressed as

$$
\hat{Y}_{m, n}^{P}=\hat{C}_{m, n}^{P}+W_{m, n}
$$

where $W_{n, m}$ is the corresponding AWGN term of the output of demodulation with zero mean and variance $\sigma^{2} / 2$ in real and imaginary components.

When the AWGN term $W_{m, n}$ is neglected, we can rewrite (32) as

$$
\hat{Y}_{m, n}^{P}=e^{j \theta_{m}}= \begin{cases}e^{j \pi / 4}, & c_{m}=1 \\ e^{-j \pi / 4}, & c_{m}=2 \\ e^{j 3 \pi / 4}, & c_{m}=3 \\ e^{-j 3 \pi / 4}, & c_{m}=4\end{cases}
$$

It can be seen that the received symbols $\left\{\hat{Y}_{m, n}^{P}, n \in J_{P}\right\}$ on SI subcarriers have different phase rotation $\theta_{m}$ for different SI index $c_{m}$. The relationship between $\theta_{m}$ and $c_{m}$ of the selected signal $s_{m}^{\left(c_{m}\right)}(t)$ of the $m$ th block is listed in Table 1. 
Table 1. The relationship between phase rotation and SI index of the selected signal

\begin{tabular}{|c|c|c|}
\hline SI index $c_{m}$ & Phase rotation $\theta_{m}$ & Selected signal $s_{m}^{\left(c_{m}\right)}(t)$ \\
\hline \hline 1 & $\pi / 4$ & $S_{m}^{(1)}(t)$ \\
\hline 2 & $-\pi / 4$ & $s_{m}^{(2)}(t)$ \\
\hline 3 & $3 \pi / 4$ & $S_{m}^{(3)}(t)$ \\
\hline 4 & $-3 \pi / 4$ & $S_{m}^{(4)}(t)$ \\
\hline
\end{tabular}

According to (33), a reliable SI detector is presented by measuring the phase rotation of the embedding SI symbols. The block diagram of the proposed SI detector at the receiver is shown in Fig. 4. The proposed SI detector can obtain good performance and the probability of correct SI detection $\operatorname{Pr}\left\{\hat{c}_{m}=C_{m}\right\}$ is closed to 1 . The details of the probability of correct SI detection can be found in the Appendix.

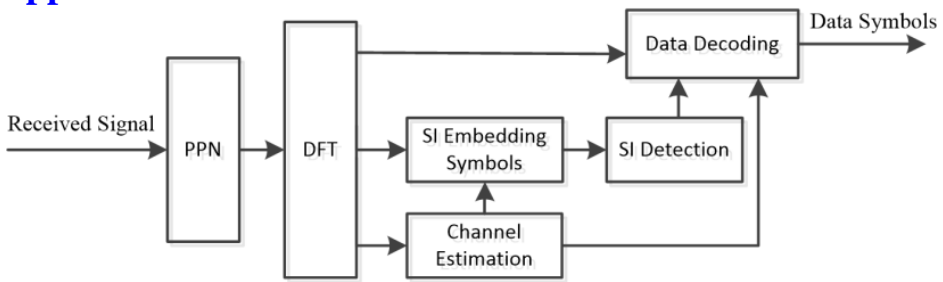

Fig 4. Block diagram of the proposed SI detector at the receiver

For an unknown SI index $c_{m}$ at the receiver, the phase estimation $\theta_{m}$, denoted by $\hat{\theta}_{m}$ of the $m$ th transmitted signal, can be calculate by

$$
\hat{\theta}_{m}=\angle \sum_{k=0}^{N_{P}} \hat{Y}_{m, n}^{P}
$$

When AWGN noise is considered, the SI index $c_{m}$ can be estimated according to Table $\mathbf{1}$ as follows

$$
\hat{c}_{m}=\left\{\begin{array}{l}
1, \quad 0<\hat{\theta}_{m} \leq \pi / 2 \\
2, \quad-\pi / 2<\hat{\theta}_{m} \leq 0 \\
3, \pi / 2<\hat{\theta}_{m} \leq \pi \\
4, \quad-\pi<\hat{\theta}_{m} \leq-\pi / 2
\end{array}\right.
$$

After $\hat{c}_{m}$ being determined, the transmitted data symbols of the $m$ th block can be correctly demodulated according to (22).

\subsection{Effects of SI Error Rate on the BER}

Let $\alpha=\operatorname{Pr}\left\{\hat{c}_{m} \neq c_{m}\right\}$ be the error rate of SI detection for the proposed CS-TR method. The BER $P_{e}$ of the proposed scheme is given as

$$
P_{e}=\alpha P_{b}^{\left(\hat{c}_{m} \neq c_{m}\right)}+(1-\alpha) P_{b}^{\left(\hat{c}_{m}=c_{m}\right)}
$$

where $P_{b}^{\left(\hat{c}_{m} \neq c_{m}\right)}$ is the conditional BER with incorrect SI detection and $P_{b}^{\left(\hat{c}_{m}=c_{m}\right)}$ is the conditional BER with correct SI detection of the proposed scheme.

In the Appendix, it can be seen that $\alpha=1-\operatorname{Pr}\left\{\hat{c}_{m}=c_{m}\right\} \approx e^{-N_{p} / 2 \sigma^{2}}$. This means that, for a given SNR, $\alpha$ exponentially decreases with the number of SI subcarriers $N_{P}$ increasing. Consequently, when $N_{P} / 2 \sigma^{2}$ is much larger than $1, \alpha$ approximately converges to zero and 
$P_{e}$ in (36) approaches to $P_{b}^{\left(\hat{c}_{m}=c_{m}\right)}$. Obviously, for $N_{P} / 2 \sigma^{2}>>1$, the proposed scheme with detected SI can obtain almost the same BER performance compared to the one with ideal SI.

\section{Computational Complexity Analysis}

Computational complexity is evaluated in terms of the number of real multiplications (RMs) required for all $M$ data blocks in one frame. For fair comparison, we assume that all schemes use the same IDFT-PPN structure for FBMC modulation and demodulation. The number of RMs of one $N$-point IDFT/DFT is $4 \times(N / 2) \log _{2} N$ where the factor 4 denotes the number of RMs per one complex multiplication. The number of RMs of one PPN is $2 \times K N$, because the PPN input is complex-valued samples with the length of $K N$ where the sample of prototype filter $p(t)$ is real-valued.

\subsection{Complexity of PAPR Reduction}

In the proposed CS-TR scheme at the transmitter in Fig. 3, two IDFTs and four PPNs are used for one data block to generate four candidate signals. Accordingly, the number of RMs for one block is $\left(2 \times 2 N \log _{2} N+4 \times 2 K N\right)$. For time shift $T / 2$ of the IDFT output, it does not require extra RM. Therefore, $M\left(4 N \log _{2} N+8 K N\right)$ RMs are required for $M$ data blocks. For TR procedure, we use two DFTs and two PPNs to demodulate the clipped signal, and two IDFTs and two PPNs to generate the TR signal for one block. Hence, the number of RMs is $4 M\left(2 N \log _{2} N+2 K N\right)$ in one frame. For the optimal factor $\rho_{m}^{(i)}$ in (29), the number of RMs required is about $2 K$ for one block where $K$ is the number of samples in $S_{m}$. Given the number of iteration $I$, the number of RMs required is $4 M I\left(2 N \log _{2} N+2 K N\right)+2 M K I$ in one frame.

Hence, the proposed scheme requires $M\left(4 N \log _{2} N+8 K N\right)+4 M I\left(2 N \log _{2} N+2 K N\right)+2 M K I$ RMs in one frame at the transmitter. Complexity comparison of PAPR reduction for original FBMC signals, CC-TR [25], OS-TR [28], SI embedded LP-FBMC [33] and the proposed CS-TR are shown in Table 2.

Table 2. Complexity comparison of different PAPR schemes at the transmitter

\begin{tabular}{|c|c|c|}
\hline Scheme & Major calculations of one data block & $\begin{array}{c}\text { Number of RMs for } M \text { data } \\
\text { blocks }\end{array}$ \\
\hline Original signal & $2 \times$ IDFTs and $2 \times \mathrm{PPNs}$ & $M\left(4 N \log _{2} N+4 K N\right)$ \\
\hline CS-TR & $\begin{array}{l}\text { candidate signals: } 2 \times \text { IDFTs and } 4 \times \text { PPNs; } \\
\text { one iteration for TR: } 2 \times \text { DFTs, } 2 \times \text { IDFTs and } \\
4 \times \text { PPNs }\end{array}$ & $\begin{array}{l}M\left(4 N \log _{2} N+8 K N\right)+ \\
4 M I\left(2 N \log _{2} N+2 K N\right)+2 M K I\end{array}$ \\
\hline OS-TR & $\begin{array}{l}\text { original signal: } 2 \times \text { IDFTs and } 2 \times \text { PPNs; } \\
\text { one iteration for TR: } 2 \times \text { DFTs, } 2 \times \text { IDFTs and } \\
4 \times \text { PPNs }\end{array}$ & $\begin{array}{l}M\left(4 N \log _{2} N+4 K N\right)+ \\
4 M I\left(2 N \log _{2} N+2 K N\right)+2 M K I\end{array}$ \\
\hline CC-TR & $\begin{array}{l}\text { original signal: } 2 \times \text { IDFTs and } 2 \times \mathrm{PPN} \text {; } \\
\text { one iteration for TR: } 2 \times \text { DFTs, } 2 \times \mathrm{IDFT} \text { and } \\
4 \times \mathrm{PPNs}\end{array}$ & $\begin{array}{l}M\left(4 N \log _{2} N+4 K N\right)+ \\
4 M I\left(2 N \log _{2} N+2 K N\right)\end{array}$ \\
\hline SI LP-FBMC & $\begin{array}{l}\text { candidate signals: } 1 \times \text { DFT, } 1 \times \text { IDFT and } \\
8 \times \text { PPN; } \\
\text { SI embedding: } 3 \times \text { phase rotations of length }-4 N \\
\text { vector }\end{array}$ & $M\left(10 N \log _{2} N+64 K N+44 N\right)$ \\
\hline
\end{tabular}

From Table 2, it can be seen that the required RMs of CS-TR, OS-TR and CC-TR methods 
vary with iterative numbers $I$ at the transmitter. For the same $I$, the complexity of the proposed scheme is slightly higher than that of OS-TR. This is because the proposed method needs extra two PPNs to generate four candidate signals.

\subsection{Complexity of SI Detection}

At the receiver, the received signal in time domain is demodulated by two PPNs and two DFTs for one block. If we omit the complexity of channel equalization procedure, for all $M$ blocks in one frame, the number of RMs required is $M\left(4 N \log _{2} N+4 K N\right)$ for basic FBMC demodulation.

For the proposed SI detector, it does not require additional RM in (34) because only the phase rotation of the summation of all $N_{P}$ SI subcarriers is detected. For SI embedded LP-FBMC [33], the SI of each block is detected by calculating the fourth power operation of $\bar{N}_{P}\left(\bar{N}_{P} \gg N_{P}\right)$ SI subcarrier and it needs $8 M \bar{N}_{P}$ RMs for all $M$ blocks. The details of complexity between our proposed CS-TR and LP-FBMC with SI detection at the receiver are shown in Table 3.

Table 3. Complexity comparison of different schemes with SI detection at the receiver

\begin{tabular}{|c|l|c|}
\hline Scheme & Major calculations of one data block & Number of RMs for $\boldsymbol{M}$ data blocks \\
\hline \hline CS-TR & $2 \times$ PPNs and $2 \times$ DFTs & $M\left(4 N \log _{2} N+4 K N\right)$ \\
\hline SI LP-FBMC & $\begin{array}{l}2 \times \text { PPNs, } 2 \times \text { DFTs and } \bar{N}_{P} \times \text { fourth power } \\
\text { operations }\end{array}$ & $M\left(4 N \log _{2} N+4 K N\right)+8 M \bar{N}_{P}$ \\
\hline
\end{tabular}

Fig. 5 shows the total complexity of OS-TR, SI embedded LP-FBMC and proposed CS-TR methods with number of total subcarriers $N$ for blocks $M=16$ and overlapping factor $K=4$. In the simulation, the number of iteration is $I=2$ for both OS-TR and CS-TR, and SI subcarriers $\bar{N}_{P}$ for LP-FBMC is $20 \%$ of total number of subcarriers $N$. It can be seen that, for the same $I$, the proposed method requires slightly higher complexity than OS-TR because of SI detection processing, while the complexity of CS-TR is lower than that of LP-FBMC because the SI detection of LP-FBMC is required to calculate the fourth power operation of all $\bar{N}_{P}$ SI subcarriers. In fact, the exact complexity of the proposed method mainly depends on the number of iteration $I$. It can be found in Section 5 based on simulation results that the proposed method requires small $I$ to achieve better PAPR performance compared to OS-TR.

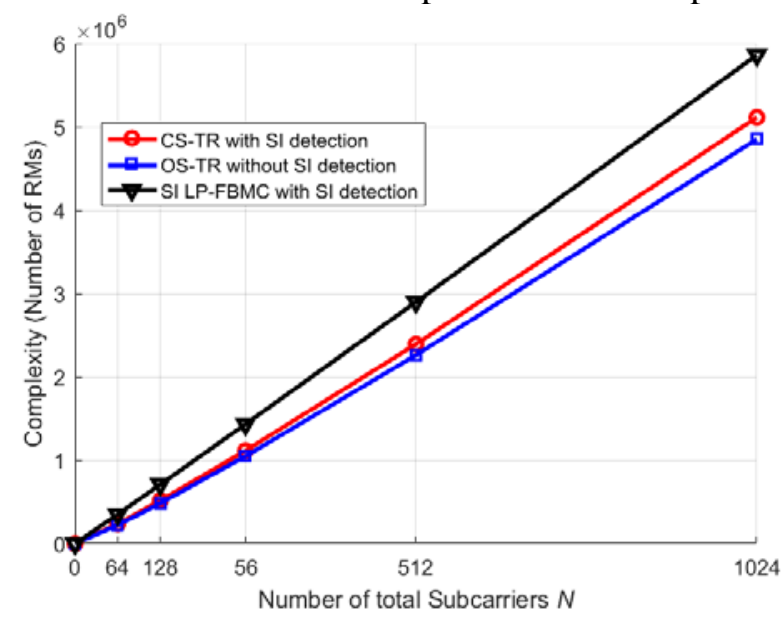

Fig 5. Complexity comparison of different schemes (Data blocks $M=16$, overlapping factor $K=4$, number of iteration $I=2$ for CS-TR and OS-TR and SI subcarriers $\bar{N}_{P}=0.2 \mathrm{~N}$ for LP-FBMC) 


\section{Simulation Results and Analysis}

Simulations are conducted to verify the effectiveness of the proposed CS-TR method in FBMC systems. The FBMC system has $N=128$ total subcarriers and the subcarrier spacing is set to $15 \mathrm{kHz}$. For the purpose of SI detection, the number of SI subcarriers is $N_{P}=4$ with QPSK constellation. The frequency selective Rayleigh fading channel, called Pedestrian-A channel [33][37], is employed for BER performance evaluation. There are $M=16$ data blocks randomly generated in a frame with 16QAM and the PAPR observation interval is set to be $T$. We adopt PHYDYAS filter [34] as the prototype filter $p(t)$ and the length of $p(t)$ is $K T$ with overlapping factor $K=4$. For comparison, CC-TR [25], OS-TR [28] and SI LP-FBMC [33] are considered under a common IDFT-PPN structure.

Both PAPR reduction and BER performances are assessed by simulations. We use complementary cumulative density function (CCDF) of PAPR to evaluate the PAPR reduction performance. The CCDF of PAPR denoted by $\operatorname{Pr}\left\{P A P R \geq P A P R_{0}\right\}$ is defined as the probability that the PAPR of FBMC signal exceeds a given threshold $P A P R_{0}$. To evaluating the BER performance with considering the impact of non-linear distortion of $\mathrm{PA}$, we adopt a solid-state power amplifier (SSPA) in [38] with input back-off (IBO) at the transmitter. In the simulation, the number of PRTs $N_{R}$ is random selection and the CR for TR is $\lambda=6 \mathrm{~dB}$.

Fig. 6 shows the PAPR reduction performance of the proposed CS-TR scheme with the number of SI subcarriers $N_{P}=4$ when the number of iteration is $I=2$. The numbers of PRTs are $N_{R}=4,8,12$ and 16, respectively. As shown in Fig. 6, the PAPR reduction performance of the proposed method is improved as $N_{R}$ increasing from $N_{R}=4$ to $N_{R}=12$. However, the PAPR performance is almost the same for $N_{R}=12$ and 16 . This is because the proposed cycle shift processing can reduce PAPR of original FBMC signal. Moreover, the TR weighted by optimal factor method further reduces the PAPR with fast convergence speed. Therefore, the number of PRTs $N_{R}=12$ is selected in the following simulations.

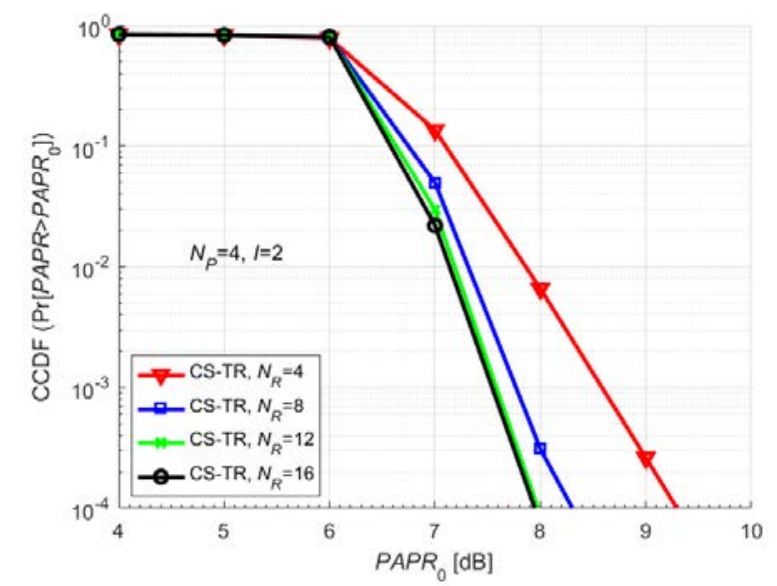

Fig 6. PAPR performance of the proposed CS-TR scheme with different numbers of PRTs $N_{R}$ (SI subcarriers $N_{P}=4$ and number of iteration $I=2$ )

Fig. 7 gives the PAPR reduction performance of the proposed CS-TR scheme with different numbers of iteration $I$. The CCDF curves of original FBMC signal and proposed CS-TR scheme with $I=0$ are also included for comparison. For the proposed scheme with $I=0$ situation, it means that the PAPR performance is obtained only by the selected signal based on cycle shift processing without iteration. We can observe that the proposed scheme achieves significantly PAPR reduction performance compared with original FBMC signal. For instance, 
at $\operatorname{Pr}\left\{P A P R \geq P A P R_{0}\right\}=10^{-4}$, compared with original FBMC signal, the proposed method achieves about $1.5 \mathrm{~dB}, 3.2 \mathrm{~dB}, 4.2 \mathrm{~dB}, 4.6 \mathrm{~dB}$, and $4.9 \mathrm{~dB}$ PAPR reduction for $I=0,1,2,3$ and 4 , respectively. It also can be seen that the proposed scheme improves PAPR performance with $I$ increasing. However, when $I>2$, the PAPR performance gap of the proposed scheme becomes small. From Table 2, we can see that the computational complexity is proportional to $I$. Therefore, it is a tradeoff between the PAPR reduction and the number of iteration $I$.

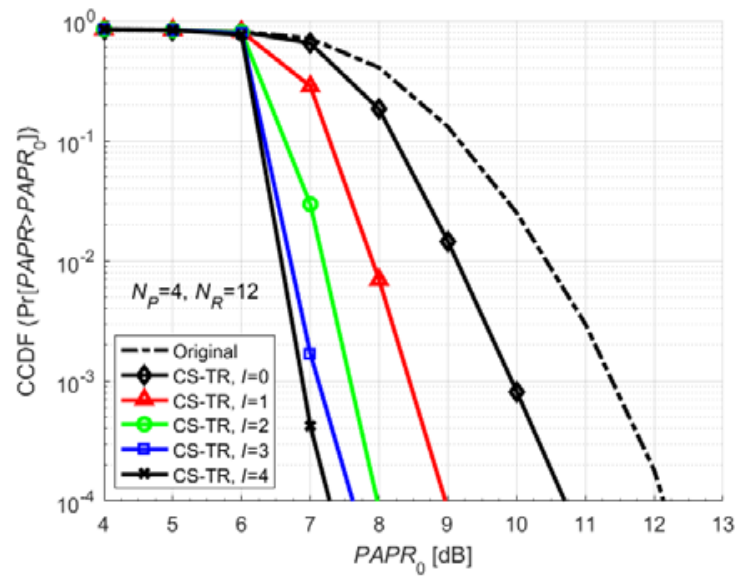

Fig 7. PAPR performance of the proposed CS-TR scheme with different numbers of iteration $I$ (SI subcarriers $N_{P}=4$ and number of PRTs $N_{R}=12$ )

Fig. 8 illustrates the PAPR performance comparison of CC-TR, OS-TR, SI LP-FBMC and the proposed CS-TR schemes. For TR iteration processing, the number of iteration $I=2$ and 4 are used to CC-TR, OS-TR and CS-TR, respectively. For fair comparison with the same data transmission subcarriers, the number of SI subcarriers $N_{P}=4$ and the number of PRTs $N_{R}=12$ are used in CS-TR while $N_{R}=16$ is used in CC-TR and OS-TR. In the simulation, the clipping ratio is set $\lambda=6 \mathrm{~dB}$ and data blocks with 16QAM are used. As shown in Fig. 8, the PAPR performance of CC-TR, OS-TR and CS-TR improves from $I=2$ to $I=4$. For the same number of iteration $I$, the proposed scheme can achieve the best PAPR reduction performance among the four schemes. This is because the proposed scheme is a joint optimization method by using cycle shift and weighted TR procedure to reduce the PAPR of FBMC signals. However, the proposed scheme achieves slightly higher PAPR performance than OS-TR for $I=4$.

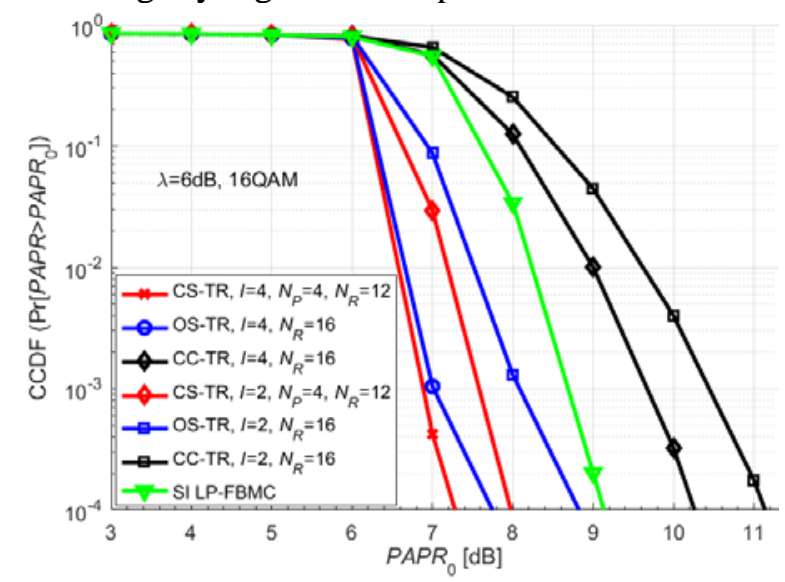

Fig 8. PAPR performance comparison of different schemes with different numbers of SI subcarriers $N_{P}$ and PRTs $N_{R}$ (Clipping ratio $\lambda=6 \mathrm{~dB}$ and data blocks with 16QAM) 
Fig. 9 depicts the SI error rate (SIER) performance comparison with different numbers of SI subcarriers $N_{P}$ for $\mathrm{SNR}=2 \mathrm{~dB}, 6 \mathrm{~dB}$ and $12 \mathrm{~dB}$, respectively. In the simulation, the IBO of SSPA is set $4 \mathrm{~dB}$ and data blocks with 16QAM are used. As we can see, for a given SNR, the proposed CS-TR method can achieve much better SIER performance than SI embedded LP-FBMC when $N_{P}$ increases. Moreover, for a given $N_{P}$, the SIER performance of the proposed method is improved with SNR increasing. In addition, for a small $N_{P}$, the proposed SI detector can obtain a good SIER performance while the SI detector of LP-FBMC is invalid.

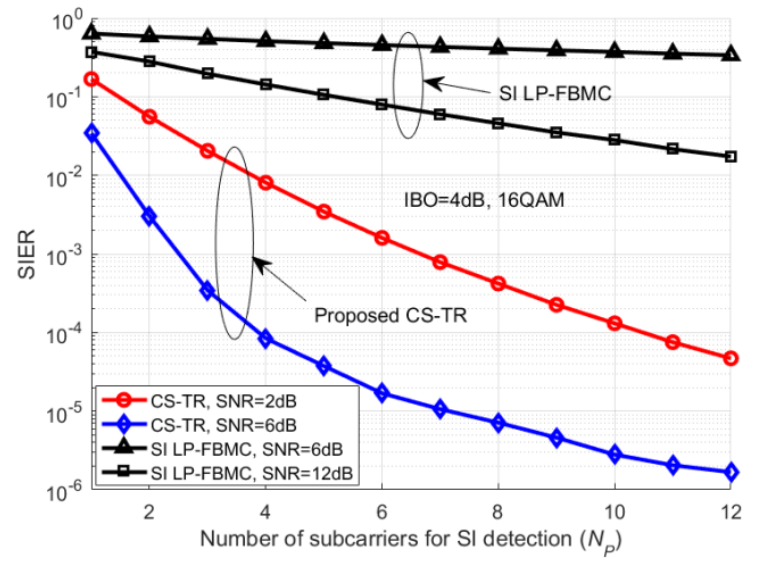

Fig 9. SIER performance comparison with different numbers of SI subcarriers $N_{P}(\mathrm{IBO}=4 \mathrm{~dB}$ of SSPA and data blocks with 16QAM)

Fig. 10 shows the SIER performance comparison of the proposed CS-TR method and SI embedding LP-FBMC scheme with different SNRs. The IBO of SSPA is $4 \mathrm{~dB}$ and data blocks with 16QAM are used in the simulation. It can be observed that the proposed SI detector of CS-TR can achieve much better SIER performance than that of LP-FBMC. At low SNR region, e.g. $\mathrm{SNR}=6 \mathrm{~dB}$, the proposed SI detector can obtain satisfied SIER performance with the number of SI subcarriers $N_{P}=4$ while the SIER performance is still poor for LP-FBMC with $N_{P}=20$. It means that the proposed SI detector can achieve good SIER performance with small numbers of $N_{P}$. The reason is that the SI is determined by phase detection for adding all $N_{P}$ SI subcarriers with the same phase offset, which effectively improve the SNR of SI detection.

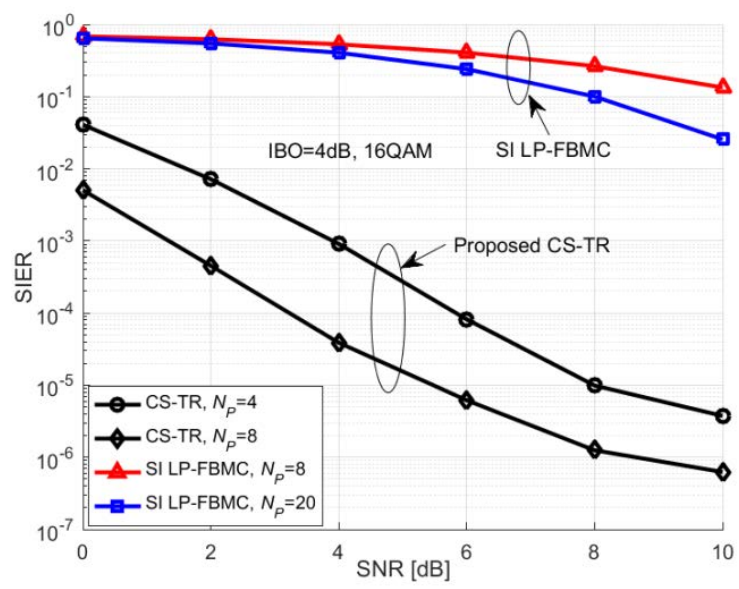

Fig 10. SIER performance comparison with different SNRs (IBO $=4 \mathrm{~dB}$ of SSPA and data blocks with 16QAM) 
Fig. 11 gives the BER performance of the proposed CS-TR scheme with different numbers of iteration $I$ under fading channel. The number of SI subcarriers is $N_{P}=4$ and the number of PRTs is $N_{R}=12$. The IBO of SSPA is $4 \mathrm{~dB}$ and data blocks with 16QAM are used. It can be seen that the proposed CS-TR method with SI detection can achieve the same BER performance compared to the one with perfect SI. As expected, the proposed scheme can improve BER performance from $I=0$ to 4 . In addition, the BER performance gap of the proposed scheme decreases with $I$ increasing. The reason is that, for a fixed threshold of clipping ratio, the PAPR reduction gap of the proposed method rapidly decreases with $I$ increasing.

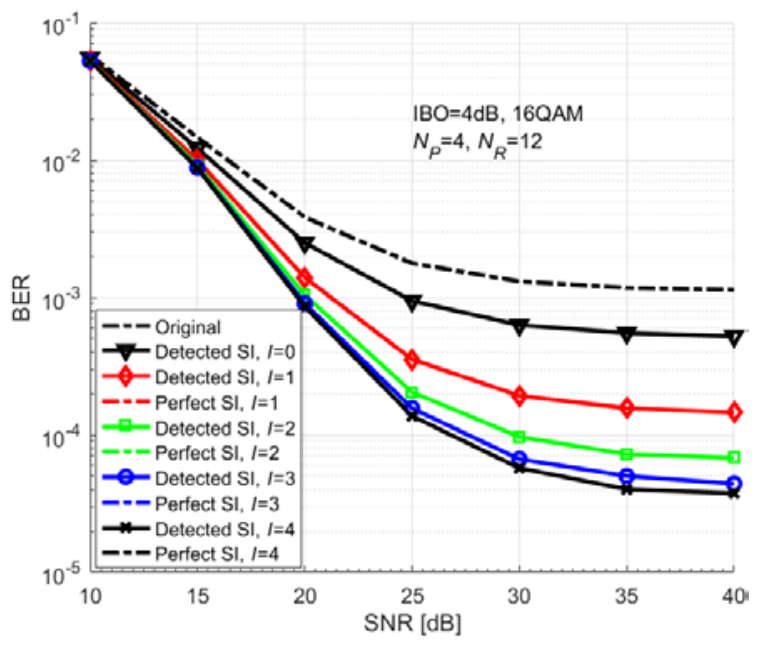

Fig 11. BER performance of the proposed CS-TR scheme with different numbers of iteration $I$ under fading channel (IBO $=4 \mathrm{~dB}$ of SSPA, SI subcarriers $N_{P}=4$ and number of PRTs $N_{R}=12$ )

Fig. 12 shows the BER performance comparison for original FBMC signal, CC-TR, OS-TR, SI embedding LP-FBMC and the proposed CS-TR schemes under fading channel. During the simulation, the IBO of SSPA is $4 \mathrm{~dB}$ and data blocks with 16QAM are used. The number of PRTs is $N_{R}=16$ for both CC-TR and OS-TR while $N_{R}=12$ for the proposed CS-TR method. For SI detection, the numbers of SI subcarriers are $N_{P}=4$ for CS-TR and $N_{P}=20$ for LP-FBMC. It is shown that, for a given value of $I$, the proposed CS-TR scheme has the best BER performance among four methods. It can be observed that the proposed CS-TR scheme with SI detection can obtain almost the same BER performance from $S N R=10$ to $40 \mathrm{~dB}$ compared to the theoretical analysis in (36). Moreover, the BER performance of the proposed method with $N_{P}=4$ is better than that of LP-FBMC with $N_{P}=20$. The reason is that the proposed method can achieve better PAPR reduction and SIER performances than LP-FBMC. It also can be seen that the BER performance of the proposed method is better than that of OS-TR when $I=1$ and 2 . This is due to the fact that our proposed scheme can effectively improve the PAPR performance shown in Fig. 8, which will lead to lower non-linear distortion when the transmitted signal with 16QAM passes through SSPA. 


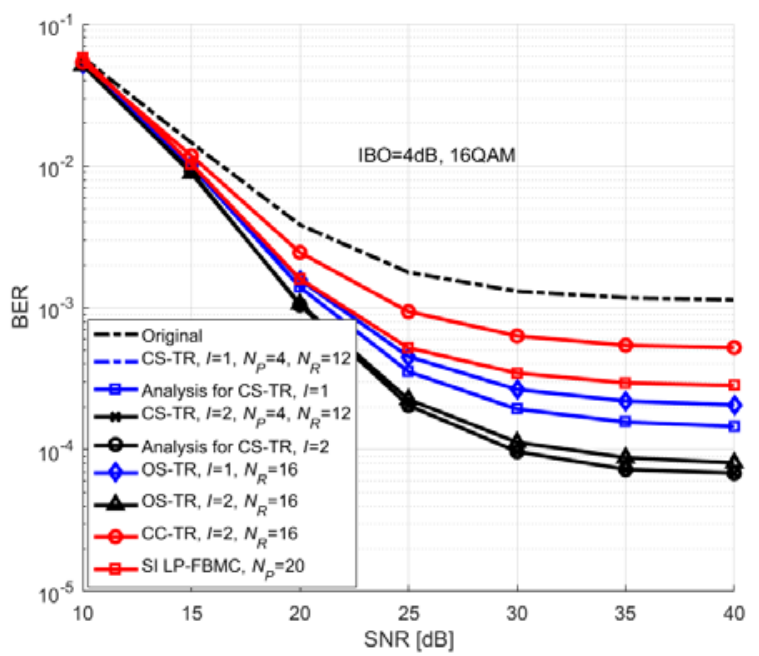

Fig 12. BER performance comparison of different schemes under fading channel (IBO=4dB of SSPA and data blocks with 16QAM)

\section{Conclusion}

In this paper, a new CS-TR scheme using joint optimization based on candidate signals selection and iterative TR was proposed to reduce the PAPR of FBMC/OQAM signals. At the transmitter, the proposed method selected the signal with the minimum PAPR from candidate signals based on cyclic shift of IDFT and embeds the SI of selected signal in sparse symbols with QPSK modulation. Furthermore, the TR with optimal scaling factor method was employed for the selected signal with fast convergence. At the receiver, the SI could be easily determined by using phase rotation detection method. Simulation results showed that the proposed method was able to achieve satisfied PAPR and BER performance for conventional FBMC systems. Therefore, the proposed CS-TR scheme was attractive for practical FBMC systems, especially for those with high order modulation.

\section{Appendix}

The received symbol $\hat{Y}_{m, n}^{P}=\hat{C}_{m, n}^{P}+W_{m, n}$ in (32) can be considered as a complex Gaussian random variable with mean $1 / \sqrt{2}$ and variance $\sigma^{2} / 2$ in real and imaginary components. Obviously, $\bar{X}=\sum_{k=0}^{N_{P}} \hat{Y}_{m, n}^{P}$ in (34) is a complex Gaussian random variable with mean $\mu=N_{P} / \sqrt{2}$ and variance $\bar{\sigma}^{2}=N_{P} \sigma^{2} / 2$ in both real and imaginary parts.

Without loss of generality, we assume that the SI of selected signal of the $m$ th block is $c_{m}=1$. Consequently, the probability of correct SI detection $\operatorname{Pr}\left\{\hat{c}_{m}=c_{m}\right\}$ can be given as

$$
\operatorname{Pr}\left\{\hat{c}_{m}=c_{m}\right\}=\operatorname{Pr}\left\{0<\hat{\theta}_{m} \leq \pi / 2\right\}
$$

The probability of (37) is rewritten as 


$$
\begin{aligned}
\operatorname{Pr}\left\{0 \leq \hat{\theta}_{m}<\pi / 2\right\} & =\operatorname{Pr}\{\mathfrak{R}\{\bar{X}\} \geq 0\} \times \operatorname{Pr}\{\Im\{\bar{X}\}>0\} \\
& =\left[\int_{0}^{+\infty} \frac{1}{\sqrt{2 \pi} \bar{\sigma}} e^{-(y-\mu)^{2} / 2 \bar{\sigma}^{2}} d y\right]^{2}=[1-Q(\mu / \bar{\sigma})]^{2} \\
& =1+Q^{2}\left(\sqrt{N_{P}} / \sigma\right)-2 Q\left(\sqrt{N_{P}} / \sigma\right)
\end{aligned}
$$

where $Q(x)=\int_{x}^{+\infty} \frac{1}{\sqrt{2 \pi}} e^{-z^{2} / 2} d z$.

Since $Q^{2}\left(\sqrt{N_{P}} / \sigma\right)$ is much smaller than $2 Q\left(\sqrt{N_{P}} / \sigma\right)$, the term $Q^{2}\left(\sqrt{N_{P}} / \sigma\right)$ can be ignored in (38). Based on the exponent approximation $Q(x) \approx \frac{1}{2} e^{-x^{2} / 2}, x>0$ [39], the probability of correct SI detection can be obtained as

$$
\operatorname{Pr}\left\{\hat{c}_{m}=c_{m}\right\} \approx 1-2 Q\left(\sqrt{N_{P}} / \sigma\right) \approx 1-e^{-N_{P} / 2 \sigma^{2}}
$$

Therefore, for $N_{P} / 2 \sigma^{2}>>1, \operatorname{Pr}\left\{\hat{c}_{m}=c_{m}\right\}$ is closed to 1 .

\section{References}

[1] G. Wunder, P. Jung, M. Kasparick, et al., “5GNOW: non-orthogonal, asynchronous waveforms for future mobile applications,” IEEE Communications Magazine, vol. 52, no. 2, pp. 97-105, Feb. 2014. Article (CrossRef Link)

[2] B. Farhang, “OFDM versus filter bank multicarrier,” IEEE Signal Processing Magazine, vol. 28, no. 3, pp. 92-112, May 2011. Article (CrossRef Link)

[3] P. Siohan, C. Siclet and N. Lacaille, "Analysis and design of OFDM/OQAM systems based on filterbank theory,” IEEE Transactions on signal processing, vol. 50, no. 5, pp. 1170-1183, May 2002. Article (CrossRef Link)

[4] D. Chen, Y. Tian, D. Qu, T. Jiang, “OQAM-OFDM for wireless communications in future internet of things: a survey on key technologies and challenges,” IEEE Internet of Things Journal, vol. 5, no. 5, pp. 3788-3809, Oct. 2018. Article (CrossRef Link)

[5] S. Bulusu, H. Shaiek, D. Roviras and R. Zayani, "Reduction of PAPR for FBMC-OQAM systems using dispersive SLM technique," in Proc. of International Symposium on Wireless Communications Systems, Barcelona, Spain, pp. 568-572, 27-29 Aug. 2014. Article (CrossRef Link)

[6] M. Laabidi, R. Zayani, R. Bouallegue, “A novel multi-block selective mapping scheme for PAPR reduction in FBMC/OQAM systems," in Proc. of World Congress on Information Technology and Computer Applications Congress, Hammamet, Tunisia, pp. 1-5, 11-13, Jun. 2015. Article (CrossRef Link)

[7] S. Bulusu, H. Shaiek, and D. Roviras, "Reducing the PAPR in FBMC-OQAM systems with low-latency trellis-based SLM technique,” EURASIP Journal on Advances in Signal Processing, vol. 2016, no. 1, pp. 132-142, Sep. 2016. Article (CrossRef Link)

[8] Z. Yang and T. Jiang, "Peak-to-average power ratio reduction for OFDM/OQAM signals via alternative-signal method,” IEEE Transactions on Vehicular Technology, vol. 63, no. 1, pp. 494-499, Jan. 2014. Article (CrossRef Link)

[9] A. Kumar and H. Rathore, "Modified DSLM technique for PAPR reduction in FBMC system," Radioelectronics and Communications Systems, vol. 62, no. 8, pp. 416-421, Aug. 2019. Article (CrossRef Link)

[10] Y. Xia and J. Zhang, "PAPR reduction for OFDM/OQAM signals using offset-symbols joint SLM method,” Journal of communications, vol. 11, no. 11, pp. 998-1004, Nov. 2016. Article (CrossRef Link)

[11] D. Qu, S. Lu and T. Jiang, "Multi-block joint optimization for the peak-to-average power ratio reduction of FBMC-OQAM signals,” IEEE Transactions on Signal Processing, vol. 61, no. 7, pp. 1605-1613, Apr. 2013. Article (CrossRef Link) 
[12] C. Ye, Z. Li, T. Jiang, et al., "PAPR reduction of OQAM-OFDM signals using segmental PTS scheme with low complexity,” IEEE Transactions on Broadcasting, vol. 60, no. 1, pp. 141-147, Mar. 2014. Article (CrossRef Link)

[13] J. Moon, Y. Nam, J. Kim, "PAPR reduction in the FBMC-OQAM System via segment-based optimization,” IEEE Access, vol. 6, pp. 4994-5002, Jan. 2018. Article (CrossRef Link)

[14] J. Zhao, S. Ni, Y. Gong, "Peak-to-average power ratio reduction of FBMC/OQAM signal using a joint optimization scheme,” IEEE Access, vol. 5, pp. 15810-15819, May 2017. Article (CrossRef Link)

[15] Z. He, L. Zhou, Y. Chen and X. Ling, "Low-complexity PTS scheme for PAPR reduction in FBMC-OQAM systems,” IEEE Communications Letters, vol. 22, no. 11, pp. 2322-2355, Nov. 2018. Article (CrossRef Link)

[16] S. Ni, M. Lei, M. Zhao, M. Lit, "Improved PTS technique for the PAPR-reduction of FBMC-OQAM signals,” in Proc. of IEEE Vehicular Technology Conference (VTC-Fall), Chicago, USA, pp. 1-5, 27-30 Aug. 2018. Article (CrossRef Link)

[17] K. Liu, J. Hou, P. Zhang and Y. Liu, "PAPR reduction for FBMC-OQAM systems using P-PTS scheme,” The Journal of China Universities of Posts and Telecommunications, vol. 22, no. 6, pp. 78-85, Dec. 2015. Article (CrossRef Link)

[18] H. Wang, "A hybrid PAPR reduction method based on SLM and multi-data block PTS for FBMC/OQAM systems,” Information, vol. 9, pp. 246-257, Oct. 2018. Article (CrossRef Link)

[19] S. Lv, J. Zhao, L. Yang, Q. Li, “Genetic algorithm based bilayer PTS scheme for peak-to-average power ratio reduction of FBMC/OQAM signal,” IEEE Access, vol. 8, pp. 17945-17955, Jan. 2020. Article (CrossRef Link)

[20] V. Sundeepkumar, S. Anuradha, “Adaptive clipping-based active constellation extension for PAPR reduction of OFDM/OQAM signals,” Circuits Systems and Signal Processing, vol. 36, no. 7, pp. 3034-3046, Sep. 2016. Article (CrossRef Link)

[21] Sandeepkumar, H. Minh, N. Aslam, et al., "Adaptive scaling active constellation extension scheme with fast convergence for PAPR reduction in OFDM/OQAM signals,” in Proc. of IEEE Vehicular Technology Conference (VTC-Fall), Chicago, USA, pp. 1-6, 27-30 Aug. 2018. Article (CrossRef Link)

[22] N. Neut, B. Maharaj, F. Lange, et al., "PAPR reduction in FBMC using an ACE-based linear programming optimization,” EURASIP Journal Advanced Signal Processing, no. 1, pp. 172-192, Jun. 2014. Article (CrossRef Link)

[23] V. Sandeepkumar and S. Anuradha, "Overlapped segmental active constellation extension for the PAPR reduction of the OFDM-OQAM system,” International Journal of Intelligent Systems and Applications, vol. 8, no. 4, pp. 26-32, Apr. 2016. Article (CrossRef Link)

[24] Z. Kollar, P. Horvath, "PAPR Reduction of FBMC by Clipping and its Iterative Compensation," Journal of Computer Networks and Communications, vol. 2012, no. 4, pp. 1-11, Jan. 2012. Article (CrossRef Link)

[25] M. Laabidi \& R. Bouallegue, "Three implementations of the tone reservation PAPR reduction scheme for the FBMC/OQAM system,” IET Communications, vol. 13, no. 7, pp. 918-925, Apr. 2019. Article (CrossRef Link)

[26] S. Lu, D. Qu and Y. He, "Sliding window tone reservation technique for the peak-to-average power ratio reduction of FBMC-OQAM signals,” IEEE Wireless Communications Letters, vol. 1, no. 4, pp. 268-271, Aug. 2012. Article (CrossRef Link)

[27] T. Jiang, C. Ni, C. Ye, et al., "A novel multi-block tone reservation scheme for PAPR reduction in OQAM-OFDM systems,” IEEE Transactions on Broadcasting, vol. 61, no. 4, pp. 717-722, Dec. 2015. Article (CrossRef Link)

[28] V. Sandeep and S. Anuradha, "Novel peak-to-average power ratio reduction methods for OFDM/OQAM systems,” ETRI Journal, vol. 38, no. 6, pp. 1124-1134, Dec. 2016. Article (CrossRef Link)

[29] H. Wang, X. Wang, L. Xu and W. Du, "Hybrid PAPR reduction scheme for FBMC/OQAM systems based on multi data block PTS and TR methods,” IEEE Access, vol. 4, pp. 4761-4768, Sep. 2016. Article (CrossRef Link) 
[30] S. Ren, H. Deng, X. Qian, Y. Liu, "Sparse PTS scheme based on TR schemes for PAPR reduction in FBMC-OQAM systems,” IET Communications, vol. 12, no. 14, pp. 1722-1727, Aug. 2018. Article (CrossRef Link)

[31] D. Na and K. Choi, “Low PAPR FBMC," IEEE Transactions on Wireless Communications, vol. 17, no. 1, pp. 182-193, Jan. 2018. Article (CrossRef Link)

[32] K. Choi, “Alamouti Coding for DFT Spreading-Based Low PAPR FBMC,” IEEE Transactions on Wireless Communications, vo. 18, no. 2, pp. 926-941, Feb. 2019. Article (CrossRef Link)

[33] D. Na and K. Choi, "DFT spreading-based low PAPR FBMC with embedded side information," IEEE Transactions on Communications, vo. 68, no. 3, pp. 1731-1745, Mar. 2020. Article (CrossRef Link)

[34] Project PHYDYAS, “Deliverable 5.1: Prototype filter and structure optimization,” Jan. 2009. [Online]. Available: http://www.ict-phydyas.org/userfiles/file/PHYDYAS-D5-1.pdf.

[35] V. Singh, M. Flanagan, B. Cardiff, "Generalized Least Squares Based Channel Estimation for FBMC-OQAM,” IEEE Access, vol. 7, pp. 129411-129420, Sep. 2019. Article (CrossRef Link)

[36] E. Kofidis, "Preamble-based estimation of highly frequency selective channels in FBMC/OQAM systems,” IEEE Transactions on Signal Processing, vol. 65, no. 7, 1855-1868, Apr. 2017. Article (CrossRef Link)

[37] Guidelines for Evaluation of Radio Transmission Technologies for IMT-2000, document ITU-R M.1225, 1997.

[38] E. Dalakta, A. Dweik, A. Hazmi, et al., "Efficient BER reduction technique for nonlinear OFDM transmission using distortion prediction,” IEEE Transactions on Vehicular Technology, vol. 61, no. 5, pp. 2330-2336, Jun. 2012. Article (CrossRef Link)

[39] M. Chiani, D. Dardari and M. Simon, "New exponential bounds and approximations for the computation of error probability in fading channels," IEEE Transactions on Wireless Communications, vol. 4, no. 2, pp. 840-845, Jul. 2003. Article (CrossRef Link) 

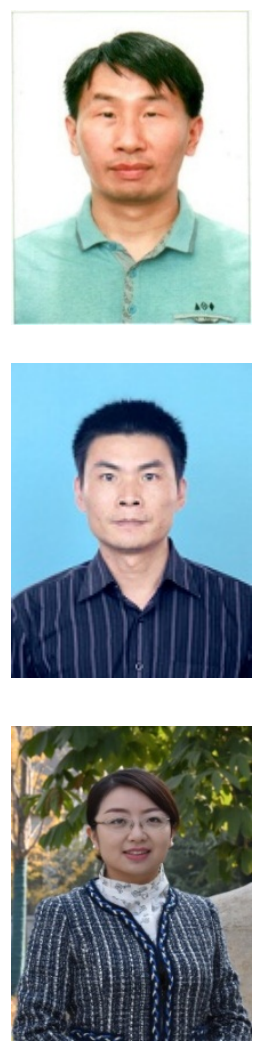

Yongpeng Shi received the B.S. degree in electronic information science from Shaanxi Normal University, Xi'an, China, in 2001. He received his M.S. and Ph.D. degree in computer science from Xidian University, Xi'an, China, in 2008 and 2018, respectively. He has been working in Luoyang Normal University since 2001. He has published around 10 peer-reviewed papers in many high quality publications, including prestigious IEEE journals and conferences. His research interests cover the next generation wireless communications, space-air-ground integrated networks and cloud computing.

Yujie Xia received the B.S. degree in electronic information engineering from Henan Normal University, Xinxiang, China, in 2001 and M.S. degree in communication and information systems from Harbin Engineering University, Harbin, China, in 2004. He received his Ph.D. degree in communication and information systems from Xidian University, Xi'an, China, in 2014. Since 2004, He has been with the Luoyang Normal University, Luoyang, China. His research interests are in the area of the communication signal processing and mobile edge computing.

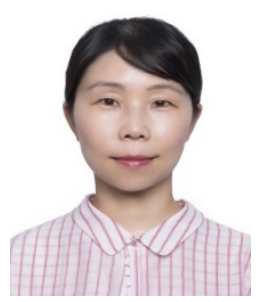

Jianhua Cui received the B.S. degree in electronic engineering and M.S. degree in circuits and systems from Zhengzhou University, Zhengzhou, China, in 2003 and 2006, respectively. She received her Ph.D. degree in information and communication engineering from National Digital Switching System Engineering \& Technological Research Center, Zhengzhou, China, in 2017. She has been working as a teacher in School of Physics and Electronic Information, Luoyang Normal University since 2006. Her research interests include the next generation wireless communications and communication signal processing. 